\title{
Lung Ultrasound in The Elderly Patients with Dyspnea: A Series Case Reports
}

\author{
Natalia Buda ${ }^{1 *}$, Krystian Sporysz ${ }^{2}$, Paweł Michalski ${ }^{2}$ and Zbigniew Zdrojewski ${ }^{1}$ \\ ${ }^{1}$ Department of Internal Medicine, Connective Tissue Diseases and Geriatrics, Medical University of Gdansk, Poland \\ ${ }^{2}$ Student scientific circle at clinic of Internal Medicine, Connective Tissue Diseases and Geriatrics, Medical University of Gdansk, Poland
}

Received: 制: September 26, 2018; Published: 制 September 28, 2018

*Corresponding author: Natalia Buda, Department of Internal Medicine, Connective Tissue Diseases and Geriatrics, Medical University of Gdansk, Poland

Abstract

Dyspnea is a common symptom and leading cause of hospital admissions of geriatric patients. Elderly patients who need hospitalisation, characterized by multiple chronic diseases. The most common causes of dyspnea are deterioration of Chronic Heart Failure (CHF) and pneumonia. Basic diagnostic tools for dyspnea such as a chest X-ray, ECG and laboratory testing (including B-type natriuretic peptide marking) often fail in helping us reach a diagnosis. A transthoracic Lung Ultrasound (LUS) appears to be a helpful, safe and simple diagnostic method.

Keywords: Dyspnea; Pneumonia; Lung Ultrasound; Pulmonary Edema

\section{Introduction}

Dyspnea is a common symptom found in millions of patients with various lung diseases. One of the most frequently encountered reasons for the admission of elderly patients is a lower respiratory tract infection alongside a chronic respiratory and/or circulatory system disease. The multitude of coexisting conditions, especially in the lungs, may hinder the identification of the underlying cause of dyspnea [1]. The standard and most frequently performed diagnostic procedure is a chest X-ray. In a elderly patients with symptoms of respiratory failure and in severe general condition the usefulness of a chest X-ray is limited. The causes of that are: the limitation to only one projection (due to patient either lying down, or assuming a semi reclining position), limited sensitivity and specificity $[2,3]$. Recent years have seen numerous publications showing a high effectiveness of lung ultrasound when comparing with a chest X-ray $[4,5]$

\section{Case Report}

\section{Patient 1}

Patient, a 70-year old male, admitted to the Department on account of resting dyspnea that has been increasing for about a week. On admission the patient was confused. Non-invasive pulse oxymetry showed $85 \%$, blood pressure was $170 / 110 \mathrm{mmHg}$, heart beat was irregular, approximately $130 / \mathrm{min}$. Auscultation revealed bilateral crackles above the inferior angles of the scapula. ECG showed tachyarythmia- atrial fibrillation with ventricle heart rate 140/min. The lab tests showed elevated inflammatory markers
(CRP 78mg/dL). Indicators of heart muscle damage were low in two samples. Bedside chest X-ray concluded that "the lungs are free of inflammatory consolidations, considerable perihilar congestion of the pulmonary circulation and cor bovinum". A bedside lung ultrasound showed numerous B-line artifacts forming the so called alveolar-interstitial syndrome" in lower and medial lung fields in both lungs- typical for pulmonary edema, and a consolidation in the lower lobe of the left lung with a dynamic air bronchogram corresponding with inflammatory symptoms (Figures 1a-3a).

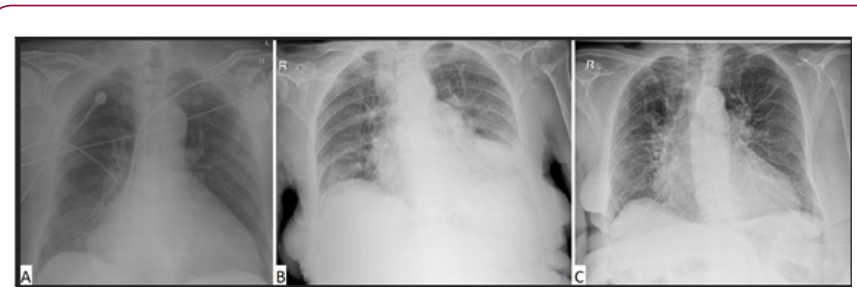

Figure 1: A bedside chest X-Ray. A- Patient 1, B- Patient 2, C- Patient 3. All X Ray shows congestion.

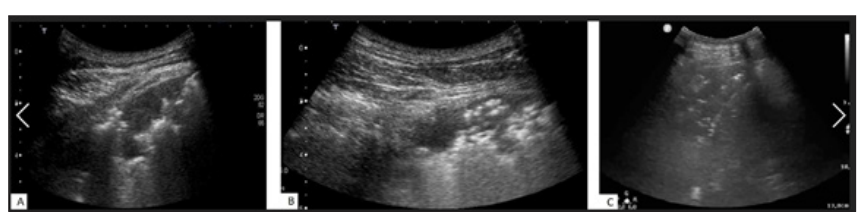

Figure 2: An image of subpleural lung consolidation using lung ultrasound. A- Patient 1, B- Patient 2, C- Patient 3. 


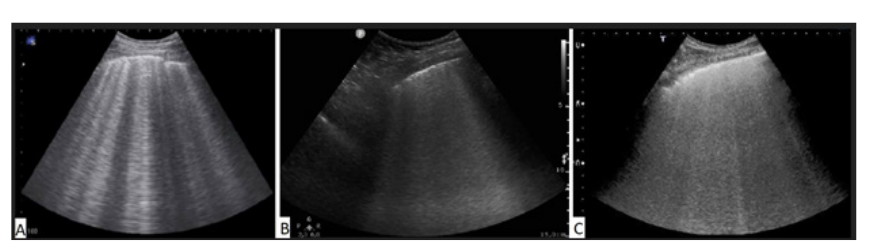

Figure 3: An image of multiple B-line forming alveolarinterstitial syndrome using lung ultrasound. A- Patient 1 , B- Patient 2, C-Patient 3.

\section{Patient 2}

Patient, an 88-year, admitted to the Department on account of increasing dyspnea (NYHA IV) developing over the course of two weeks, and swelling of the shanks. On admission the patient was in a serious condition, conscious, logically and verbally coherent. Examination revealed crackles at the bases of both lungs, enlarged liver, substantial shank and sacral area edema, BP $110 / 60 \mathrm{mmHg}$, Sp02 88\%. Lab tests showed, elevated inflammatory markers, elevated BNP levels, abnormal renal function markers. Chest X-ray description: the visible lung zones are free of consolidations; signs of congestion are visible in the perihilar area. Lung ultrasound: bilateral numerous B-line artifacts forming alveolar-interstitial syndrome to the level of lateral margin of the scapula, an image corresponding with pulmonary edema. In the zone of the lower lobe of the right lung a visible consolidation with a dynamic air bronchogram- inflammatory lesions (Figures 1b-3b).

\section{Patient 3}

Patient, a 91-year, was admitted to the Department on account of chronic dyspnea, which worsened significantly a day before admission, alongside a feeling of compression in the chest. On admission the patient was in a fair general condition. Examination revealed crackles at the bases of both lungs, Sp02 94\%, BP 11/80 $\mathrm{mmHg}$, heart beat irregular, around 134/min. Lab tests showed: abnormal renal function parameters, elevated BNP and heart damage markers. ECG revealed atrial fibrillation with a rapid ventricle beat of $140 / \mathrm{min}$. Chest X-ray description: congestion and slight interstitial edema, with Kerley B lines in phrenicocostal angles; the lungs without consolidations. The ultrasound showed bilaterally visible B-line artifacts, forming alveolar-interstitial syndrome from the bases of the lungs to the third intercoastal areasigns of pulmonary edema. Additionally, in the lower zone of the left lung a consolidation with a dynamic air bronchogram- signs of inflammatory lesions (Figures 1c-3c).

\section{Discussion}

All of the described patients have had chest X-rays in the AP projection, two of them also had them in the lateral projection, all described by a radiology specialist. All of the patients showed congestion in the pulmonary circulation of varying degree and an enlarged silhouette of the heart. None of the imaging's showed inflammation. The lung ultrasound that followed showed B-lines that formed alveolar-interstitial syndrome - typical for pulmonary edema in all the patients (which corresponded to the X-ray). Moreover, the ultrasound exam revealed a characteristic image of inflammatory lesions which were not seen in the X-ray. used. A lack of proper diagnosis or a delay in treatment in elderly patients with pneumonia might end fatally, the fatality of communityacquired pneumonia may be as high as 30\% [6,7]. Pneumonia symptoms in a geriatric population aren't usually specific, as is there clinical presentation, which only enhances the usefulness of lung ultrasound which can provide such information's.

\section{Conclusion}

Modern use of lung ultrasound offers a vast array of diagnostic options to an attending physician, especially within the limitations of a night shift. In all of the presented cases a bedside lung ultrasound allowed us to diagnose the complex cause of dyspnea in a few minutes. Ultrasound examinations are easy to perform and completely safe.

\section{References}

1. Teixeira A, Arrigo M, Tolppanen H, Gayat E, Laribi S, et al. (2016) Management of acute heart failure in elderly patients. Arch Cardiovasc Dis 109: $422-430$.

2. Ticinesi A, Lauretani F, Nouvenne A, Mori G, Chiussi G, et al. (2016) Lung ultrasound and chest $\mathrm{x}$-ray for detecting pneumonia in an acute geriatric ward. Medicine (Baltimore) 95(27): e4153.

3. Gargani L, Picano E (2015) The risk of cumulative radiation exposure in chest imaging and the advantage of bedside ultrasound. Crit Ultrasound J 7: 4 .

4. Alzahrani SA, Al-Salamah MA, Al-Madani WH, Elbarbary MA (2017) Systematic review and meta-analysis for the use of ultrasound versus radiology in diagnosing of pneumonia. Crit Ultrasound J 9(1): 6.

5. Volpicelli G, Elbarbary M, Blaivas M, Lichtenstein DA, Mathis G, et al. (2012) International evidence-based recommendations for point-ofcare lung ultrasound. Intensive Care Med 38: 577-591.

6. Ye X, Xiao H, Chen B, Zhang S (2015) Accuracy of lung ultrasonography versus chest radiography for the diagnosis of adult community-acquired pneumonia: Review of the literature and meta-analysis. PLOS ONE 10(6): e0130066.

7. Janssens JP, Krause KH (2004) Pneumonia in the very old. Lancet Infect Dis 4(2): 112-124. 


\section{ISSN: 2574-1241}

DOI: 10.26717/BJSTR.2018.09.001802

Natalia Buda. Biomed J Sci \& Tech Res

(c) (i) This work is licensed under Creative

Submission Link: https://biomedres.us/submit-manuscript.php

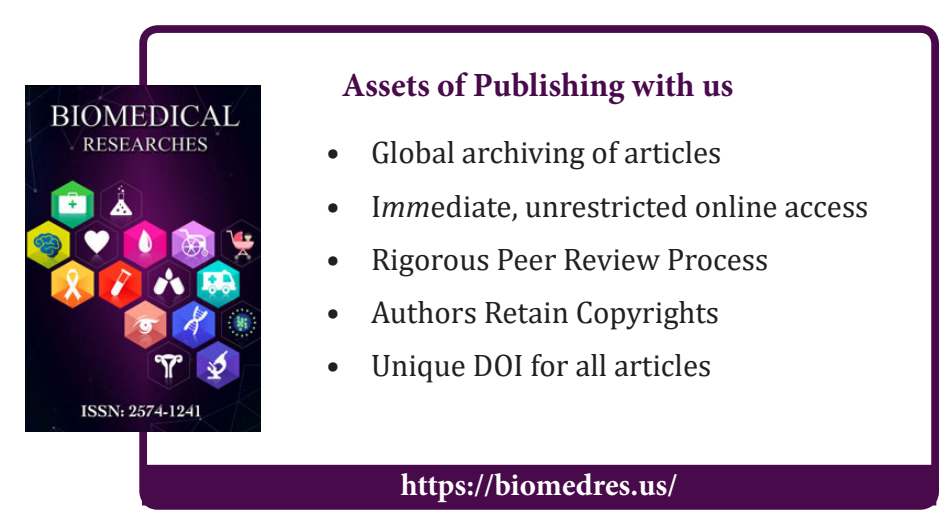

